\title{
Prognostic significance of diffuse sclerosing variant papillary thyroid carcinoma: a systematic review and meta-analysis
}

\author{
Huy Gia Vuong1,2, Tetsuo Kondo', Thong Quang Pham², Naoki Oishi', \\ Kunio Mochizuki ${ }^{1}$, Tadao Nakazawa', Lewis Hassell ${ }^{3}$ and Ryohei Katoh ${ }^{1}$ \\ 1Department of Pathology, University of Yamanashi, Yamanashi, Japan, ²Department of Pathology, Cho Ray \\ Hospital, Ho Chi Minh City, Vietnam, and ${ }^{3}$ Department of Pathology, Oklahoma University of Health Sciences \\ Center, Oklahoma City, Oklahoma, USA
}

\author{
Correspondence \\ should be addressed \\ to T Kondo \\ Email \\ ktetsuo@yamanashi.ac.jp
}

\begin{abstract}
Objective: Diffuse sclerosing variant papillary thyroid carcinoma (DSVPTC) is an uncommon variant of papillary thyroid carcinoma (PTC). The biological behaviors and prognostic outcomes of this variant, however, are still controversial. The aim of this systematic review and meta-analysis is to investigate the prognostic significance and outcomes of DSVPTCs in comparison with classical PTCs (cPTCs).

Methods: An electronic search was performed in five libraries: PubMed, Scopus, ISI, World Health Organization Global Health Library (WHO GHL) and Virtual Health Library (VHL) in June 2016. Published data were extracted and were pooled into odds ratios (OR), mean differences and corresponding $95 \%$ confidence intervals $(\mathrm{Cl})$ using random-effect model. Publication bias was analyzed using Egger's regression test and funnel plot observation.

Results: From 315 articles, we included 16 articles comprising 732 DSVPTCs for meta-analysis. Overall, DSVPTC manifested more aggressive clinicopathological behaviors than CPTC such as higher rate of vascular invasion (OR: 5.33; 95\% Cl: 3.08-9.23), extrathyroidal extension (OR: 2.96; 95\% Cl: 2.04-4.30), lymph node metastasis (OR: 5.40; 95\% Cl: 2.82-10.35), distant metastasis (OR: 3.61; 95\% Cl: 1.89-6.88) and were more likely to relapse (OR: 2.83 ; 95\% Cl: 1.59-5.05). DSVPTC patients were associated with a worsened overall survival (HR: 1.89; 95\% Cl: 1.36-2.62). Conclusion: DSVPTCs should be considered high-risk PTCs because of high propensity for tumor invasion, metastasis, relapse and mortality. Aggressiveness of DSVPTCs might be related to a different molecular pathway than that in CPTCs.
\end{abstract}

\section{Introduction}

Thyroid cancer is the most common endocrine cancer (1). Thyroid carcinoma is classified into different subtypes: papillary, follicular, poorly differentiated, undifferentiated and medullary carcinoma (2). PTC is the most common subtype and comprises more than $85 \%$ of thyroid cancer. From a morphological point of view, PTCs are subdivided into various variants with variable clinicopathological characteristics and biological behaviors (2). Most of the variants of PTC, however, do not show prognostic significance.
Only a few histological subtypes such as tall cell variant or columnar cell variant are considered aggressive variants (3).

In 1985, Vickery et al. initially described a variant of PTC showing diffuse involvement of thyroid gland (4). This rare variant was named DSVPTC in the World Health Organization (WHO) classification and was characterized by extensive squamous metaplasia, diffuse fibrosis, calcification, abundant lymphocytic infiltration and psammoma bodies (2). Because of
두 2017 European Society of Endocrinology Printed in Great Britain
Published by Bioscientifica Ltd. 
its rarity, the clinicopathological characteristics and prognostic significance of DSVPTC were not well characterized and have been continuously debated. Several studies reported that DSVPTC was associated with aggressive behaviors and poor prognosis $(5,6$, $7,8)$. These findings, however, were not confirmed in other studies $(9,10,11)$.

In this systematic review and meta-analysis, we aimed to investigate the prognostic significance, outcomes and molecular profiles of DSVPTCs in comparison with classical PTCs (cPTCs) and to overcome the limitations of small sample size of prior individual studies.

\section{Methods}

\section{Search strategy and study identification}

An electronic search in five databases (PubMed, Scopus, ISI Web of Science, WHO GHL and VHL) was performed to identify relevant articles. We used search term: ((diffuse sclerosing) AND (papillary thyroid) AND (carcinoma OR cancer OR tumor OR tumor OR neoplasm)). We included publications published from inception to August 2016. Additionally, we performed a manual search by reviewing the citations within the included publications and reviews. Our study strictly followed the recommendation of Preferred Reporting Items for Systematic Review and Meta-analysis (PRISMA) statement (Supplementary Table, see section on supplementary data given at the end of this article) (12).

\section{Selection criteria and abstract screening}

We imported search results from all libraries into Endnote (Thompson Reuters) and deleted the duplicates. Two reviewers ( $\mathrm{H} \mathrm{G} \mathrm{V}$ and T Q P) independently screened the titles and abstracts using the predetermined selection criteria. We used the following inclusion criteria: articles comparing any of clinicopathological features such as gender, age, tumor size, multifocality, lymphovascular invasion (LVI), extrathyroidal extension (ETE), lymph node metastasis (LNM), distant metastasis at the time of diagnosis (DM), recurrence/persistence (R/P) and survival outcomes of DSVPTCs and cPTCs. The exclusion criteria were (i) data sets considered as overlapping, (ii) review, (iii) case report or case series with no group of cPTCs for comparison, (iv) studies including cPTCs and other variants of PTC into comparing group and (v) conference, proceeding paper, poster, thesis, book. Discrepancies between two reviewers were solved by discussion moderated by a supervisor.

\section{Full-text screening and data extraction}

Two reviewers ( $\mathrm{H} \mathrm{G} \mathrm{V}$ and T Q P) screened full-text of potential articles and extracted data into a predefined data extraction form. Disagreements were solved by discussion by reviewers or supervisor if necessary. Following details of DSVPTC and cPTC group were extracted: institution, city, country, year of publication, time of operation, study design, age, gender, tumor size, multifocality, LVI, ETE, LNM, DM, R/P and overall survival (OS).

\section{Data analysis}

The Review Manager 5.3 software (Cochrane Collaborative, Oxford, UK) was used for statistical analysis. We estimated mean and standard deviation (s.D.) value as described previously (13). Pooled estimates of odds ratios (ORs), mean differences (MDs) and corresponding 95\% confidence intervals (CIs) were calculated using randomeffect model because this model takes into account withinstudy heterogeneity and yield the identical results as the fixed-effect model in the absence of heterogeneity (14). Matched factors between DSVPTC and cPTC groups, if present, were excluded from the analyses. Because survival rates at specific time points are known to be incompatible for meta-analyses of time-to-event data, pooled hazard ratio (HR) for OS was calculated using the random-model effect weighted by inverse variance method. HR and its 95\% CI were directly collected from the original articles or indirectly estimated from Kaplan-Meier curve using the methods by Tierney et al. (15) for studies that did not provide $\mathrm{HR}$ and its 95\% CI.

Heterogeneity between studies was quantified by the $I^{2}$ statistic. (16) Inconsistency across studies was classified as low if $25 \leq I^{2}<50 \%$ and high if $I^{2} \geq 75 \%$ (17). Publication bias was analyzed by using Egger's regression test and funnel plot to evaluate the presence of publication bias. Funnel plot and Egger's regression test was analyzed using MAVIS, version 1.1.2 - a R package (18). A $P$ value $\leq 0.05$ was considered statistically significant publication bias.

\section{Risk of bias assessment}

We assessed the methodologic quality of included studies based on the Newcastle-Ottawa Scale (NOS) for quality of 
cohort and case-control in our meta-analyses (19). Stars were awarded for each cohort and case-control study (maximum 9 stars) based on a developed checklist (19). In the second domain of outcome category for cohort studies, we awarded one star if the study had a mean or median value of follow-up period longer than five years, which was considered long enough for tumor recurrence and mortality to occur. In the last domain of outcome category for cohort studies, studies with the follow-up rate $\geq 80 \%$ or description of those lost suggesting no difference from those followed were awarded one star. Studies awarded at least six stars were considered moderate-to-high quality studies and those with a NOS value of less than six were regarded low-quality studies.

\section{Results}

We identified 315 articles after searching five libraries, merging all references into an Endnote file and deleting duplicates. After screening abstracts, only 34 articles met our criteria and all corresponding full-texts of these articles were downloaded. We excluded 18 articles after carefully reading full-texts. Finally, 16 studies with total 732 patients with DSVPTC were included for final analysis (Fig. 1) (5, 6, 7, 8, 9, 10, 11, 20, 21, 22, 23, 24, 25, $26,27,28)$. The time interval for included studies ranged from 1987 to August 2016. We describe characteristics of the 16 included studies in Table 1.

\section{Gender}

Fourteen studies, including 702 DSVPTCs and 64355 cPTCs, were compared for male proportion. Male

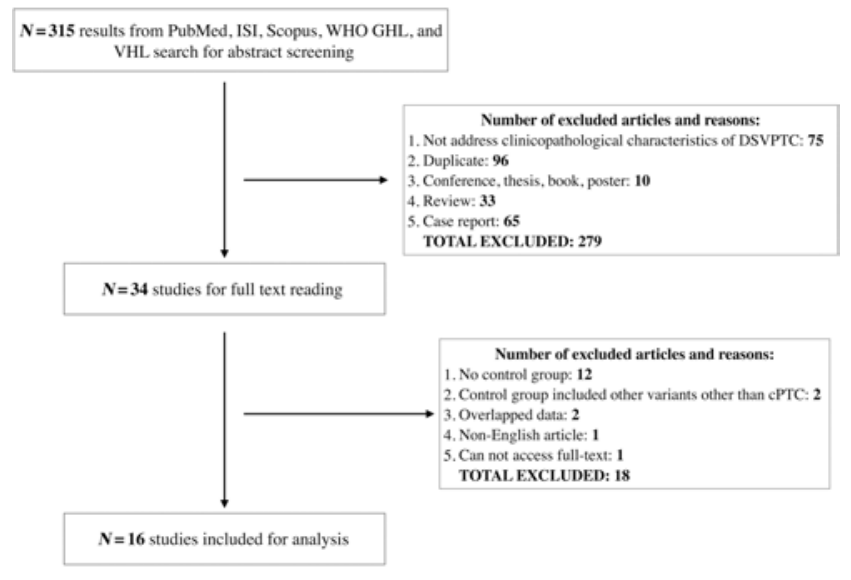

Figure 1

Flow chart of the study selection process. proportion in DSVPTC and cPTC group was $22.8 \%$ and $22.4 \%$ respectively. There was no significant difference in male proportion between DSVPTCs and cPTCs (OR: 1.15; 95\% CI: 0.77-1.73) (Supplementary Fig. 1A). Between-study heterogeneity was high $\left(I^{2}=64 \%\right.$; $P$-heterogeneity $=0.0007$ ).

\section{Age}

Mean and S.D. value of age were reported or could be estimated in seven studies, including 574 DSVPTCs and 62002 cPTCs. The mean age of DSVPTCs and cPTCs were 41.7 and 46.6 years respectively. The age of DSVPTC group was significantly younger than that of cPTC group (MD: -9.43 ; 95\% CI: -15.52 to -3.35 ) (Supplementary Fig. 1B). Amount of heterogeneity across studies was high $\left(I^{2}=94 \%\right.$; $P$-heterogeneity $\left.<0.0001\right)$.

\section{Tumor size}

We found available data of mean and S.D. value of tumor size in six studies, comprising 538 DSVPTCs and 42212 cPTCs. The mean tumor size of DSVPTCs and cPTCs were $1.80 \mathrm{~cm}$ and $1.74 \mathrm{~cm}$. There was no statistical difference in tumor size between the two variants (MD: 0.49; 95\% CI: -0.02 to 1.00) (Supplementary Fig. 1C). Among-studies heterogeneity was high $\left(I^{2}=86 \%\right.$; $P$-heterogeneity $\left.<0.0001\right)$.

\section{Multifocality}

Seven studies, including 590 DSVPTCs and 62479 cPTCs were analyzed for the difference in multifocality. The rate of multifocality was higher in DSVPTC group (35.8\%) compared to $26.0 \%$ in cPTC group. This difference, however, was not statistically significant (OR: 1.58; 95\% CI: 0.93-2.69) (Supplementary Fig. 1D). A high amount of heterogeneity across studies was found $\left(I^{2}=84 \%\right.$; $P$-heterogeneity <0.0001).

\section{Lymphovascular invasion (LVI)}

Five studies, including 158 DSVPTCs and 1057 cPTCs, were analyzed for the difference in LVI rate. The overall rate of LVI in DSVPTCs and cPTCs were $38.6 \%$ and $35.2 \%$ respectively. DSVPTCs exhibited a significantly higher propensity for LVI than cPTCs (OR: 5.33; 95\% CI: 3.08-9.23) (Supplementary Fig. 1E). Between-study heterogeneity $\mathrm{did}$ not exist $\left(I^{2}=0 \%\right.$; $P$-heterogeneity $=0.82$ ). 
Table 1 Characteristics of included studies.

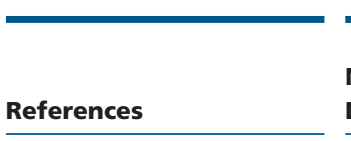

Akaishi et al. (5)

Al-Qahtani et al. (6)

Albareda et al. (9)

Balachandar et al. (20)

Falvo et al. (7)

Fridman et al. (21)

Kazaure et al. (11)

Khan et al. (22)

Kim et al. (23)

Koo et al. (28)

Nikiforov et al. (26)

Lim et al. (25)

Moreno Egea et al. (10)

85 Showed large areas of inflammatory infiltration (mainly lymphatic in nature), fibrosis, solid fibro hyaline area, psammoma bodies and squamous metaplasia. All showed the typical nuclei PTC with grooves and pseudo inclusions

11 Characterized by diffuse involvement of one or both lobes with large number of psammoma bodies, marked lymphocytic infiltration, squamous metaplasia and severe fibrosis

Regalbuto et al. (8)

245 According to the WHO classification of malignant tumors. Criteria such as the presence of numerous psammoma bodies, squamous metaplasia, marked and diffuse sclerosis were used to distinguish DSVPTC from sclerotic PTC

Soares et al. (27)

259 Diffuse involvement of one or two lobes, dense fibrosis, lymphocytic infiltration, squamous metaplasia and abundant psammoma bodies
According to the WHO classification of malignant tumors No description No description

According to the WHO classification of malignant tumors No description

According to the WHO classification of malignant tumors

No description

Papillae, empty-looking nuclei, nuclear grooves, nuclear pseudo inclusions and psammoma bodies

According to the WHO classification of malignant tumors

No description

According to the WHO classification of malignant tumors

No description

No description

Composed mainly of papillae or admixed with follicular or solid areas. Nuclei had a pale ground-glass feature, nuclear grooves pseudo inclusions. Psammoma bodies could be found

According to the WHO classification of malignant tumors

According to the WHO classification of malignant tumors

CPTC, classical papillary thyroid carcinoma; DSVPTC, diffuse sclerosing variant papillary thyroid carcinoma; PTC, papillary thyroid carcinoma; WHO, World Health Organization. 


\section{Extrathyroidal extension (ETE)}

Twelve studies, including 696 DSVPTCs and 64233 cPTCs, were compared for ETE. In total, $50.4 \%$ of DSVPTCs and $30.5 \%$ of cPTCs showed ETE. This is a statistically significant difference in the rate of ETE between the two groups (OR: 2.96; 95\% CI: 2.044.30) (Supplementary Fig. 1F). A moderate amount of heterogeneity across studies was found $\left(I^{2}=60 \%\right.$; $P$-heterogeneity $=0.005)$.

\section{Lymph node metastasis (LNM)}

In total, 491 patients with DSVPTC and 40535 patients with cPTC from 13 studies were analyzed. In DSVPTC group, $80.3 \%$ of patients manifested LNM in comparison with $48.9 \%$ in cPTC group. DSVPTCs were associated with significantly higher rate for LNM than CPTCs (OR: 5.40; 95\% CI: 2.82-10.35) (Supplementary Fig. 1G). A high degree of heterogeneity across studies was found $\left(I^{2}=77 \%\right.$; $P$-heterogeneity $<0.0001)$.

\section{Distant metastasis at the time of diagnosis (DM)}

Six studies, comprising 174 DSVPTCs and 2338 cPTCs, were included for meta-analysis. DM was found in $11.6 \%$ of DSVPTCs and in $4.2 \%$ of cPTCs. The difference in the rate of DM between DSVPTCs and CPTCs is statistically significant (OR: 3.61; 95\% CI: 1.89-6.88) (Supplementary Fig. 1H). No heterogeneity between studies was identified $\left(I^{2}=0 \%\right.$; $P$-heterogeneity $\left.=0.92\right)$. Lung is the most frequent site for DM in both groups.

\section{Recurrence/persistence of tumor (R/P)}

Ten studies, including 314 DSVPTCs and 2406 cPTCs, were included for meta-analysis. $\mathrm{R} / \mathrm{P}$ was detected in $22.0 \%$ and $10.7 \%$ of DSVPTCs and cPTCs respectively. DSVPTCs were more likely to relapse than cPTCs (OR: 2.83; 95\% CI: 1.59-5.05) (Supplementary Fig. 1I). A moderate amount of heterogeneity across studies was found $\left(I^{2}=50 \%\right.$; $P$-heterogeneity $=0.04)$.

\section{Overall survival (OS)}

In 16 included studies, we found relevant data of HR for OS or sufficient data to estimate HR for OS in only two studies $(7,11)$. We also sent emails to all corresponding authors of the included studies to ask for unreported HR and its 95\% CI for effects of DSVPTC on DSS and OS, but we received no responses. We used random-effect model weighted by inverse variance method to pool HR for OS, and the pooled result demonstrated that DSVPTC patients were associated with an increased risk of mortality compared with cPTC patients (HR: 1.89; 95\% CI: 1.36-2.62) (Supplementary Fig. 1J). No among-study heterogeneity was found $\left(I^{2}=0 \%\right)$.

\section{Quality assessment and risk of bias analysis}

Thirteen studies were retrospective cohort studies and three were case-control studies. The number of stars awarded to each of the included studies ranged from four to seven stars. Details of given stars within domain

Table 2 Newcastle-Ottawa Quality Assessment Scale for all included studies.

\begin{tabular}{l}
\hline References \\
\hline Akaishi et al. (5) \\
Al-Qahtani et al. (6) \\
Albareda et al. (9) \\
Balachandar et al. (20) \\
Falvo et al. (7) \\
Fridman et al. (21) \\
Kazaure et al. (11) \\
Khan et al. (22) \\
Kim et al. (23) \\
Koo et al. (28) \\
Lam et al. (24) \\
Lim et al. (25) \\
Moreno Egea et al. (10) \\
Nikiforov et al. (26) \\
Regalbuto et al. (8) \\
Soares et al. (27)
\end{tabular}

\begin{tabular}{l}
\hline Study design \\
\hline Case-control \\
Retrospective cohort \\
Retrospective cohort \\
Retrospective cohort \\
Case-control \\
Retrospective cohort \\
Retrospective cohort \\
Retrospective cohort \\
Case-control \\
Retrospective cohort \\
Retrospective cohort \\
Retrospective cohort \\
Retrospective cohort \\
Retrospective cohort \\
Retrospective cohort \\
Retrospective cohort \\
\hline
\end{tabular}

\begin{tabular}{ccc}
\hline \multicolumn{3}{c}{ Number of awarded stars in each domain } \\
\hline Selection & Comparability & Outcome \\
\cline { 2 - 3 } & 1 & 3 \\
3 & 0 & 3 \\
3 & 1 & 3 \\
3 & 0 & 3 \\
3 & 1 & 3 \\
3 & 0 & 2 \\
3 & 0 & 3 \\
3 & 0 & 1 \\
3 & 2 & 2 \\
3 & 0 & 3 \\
3 & 0 & 3 \\
3 & 0 & 1 \\
3 & 0 & 3 \\
3 & 0 & 2 \\
3 & 0 & 2 \\
3 & 0 & 3 \\
\hline
\end{tabular}


Table 3 Genetic backgrounds of DSVPTC and CPTC.

\begin{tabular}{|c|c|c|c|c|c|}
\hline \multicolumn{2}{|c|}{ BRAF mutations } & \multicolumn{2}{|c|}{ RETIPTC rearrangements } & \multicolumn{2}{|c|}{$A L K$ rearrangements } \\
\hline DSVPTC & CPTC & DSVPTC & cPTC & DSVPTC & cPTC \\
\hline (25): 60/98 (61.2\%) & (25): $2219 / 2947(75.3 \%)$ & & & & \\
\hline (29): $0 / 7(0 \%)$ & (29): $2 / 19(10.5 \%)$ & (32): $23 / 37(62.2 \%)$ & (34): $1 / 18(5.6 \%)$ & & \\
\hline (30): $2 / 7(28.6 \%)$ & (30): $13 / 24(54.2 \%)$ & (33): 3/7 (42.9\%) & (35): $17 / 119(14.3 \%)$ & (31): $3 / 23(13.0 \%)$ & (36): $0 / 141(0 \%)$ \\
\hline (31): 5/23 (21.7\%) & (31): $102 / 164(62.2 \%)$ & & & & \\
\hline Pooled result: $49.6 \%$ & Pooled result: $74.1 \%$ & Pooled result: $59.1 \%$ & Pooled result: $13.1 \%$ & Pooled result: $13.0 \%$ & Pooled result: $0 \%$ \\
\hline
\end{tabular}

CPTC, classical papillary thyroid carcinoma; DSVPTC, diffuse sclerosing variant papillary thyroid carcinoma.

of selection, comparability and outcome for all included studies are described in Table 2.

\section{Heterogeneity assessment and sensitivity analysis}

We used $I^{2}$ statistic to evaluate the heterogeneity across studies. In our study, heterogeneity ranged from $0 \%$ to $94 \%$. In the presence of heterogeneity, we performed sensitivity analysis by removing each of the included studies to find which studies influenced the degree of heterogeneity. All significant meta-analysis results following the leave-one-out method remained unaffected, indicating a good stability of the metaanalysis (data not shown).

\section{Publication bias}

To investigate the presence of publication bias, funnel plot of effects calculated from individual studies were performed. Funnel plots showed no strong indication of publication bias among the set of studies included in the meta-analysis. Tests of asymmetry of all estimates did not suggest any evidence of publication bias. Funnel plots and corresponding results of Egger's regression test of all estimates are presented in Supplementary Figs 2, 3, 4, 5, $6,7,8,9$ and 10 .

\section{Differences in genetic backgrounds between DSVPTC and CPTC}

Because the genetic alterations between DSVPTC and cPTC were compared in only very few studies, we did not include them for meta-analysis. We found comparable data of BRAF mutations in DSVPTC and CPTC in four studies $(25,29,30,31)$. Data for RET/PTC in DSVPTCs were separately described in two studies in Korea and Germany $(32,33)$. For comparison, we selected two Korean and German studies containing prevalence of RET/PTC rearrangements in cPTCs $(34,35)$. Prevalence of anaplastic lymphoma kinase (ALK) rearrangements in
DSVPTCs and cPTCs was only reported in an Australian and Korean study $(31,36)$. We summarize the differences in genetic backgrounds between these two PTC variants in Table 3.

\section{Discussion}

According to the 2015 American Thyroid Association (ATA) management guidelines for differentiated thyroid carcinoma, the prognostic implication of DSVPTC remains conflicting, and this variant is not considered an aggressive variant of PTC with unfavorable outcomes like tall cell, columnar cell or hobnail variants (37).

The clinicopathological features and prognostic outcomes of DSVPTCs have been summarized previously $(38,39)$. The study by Pillai et al. (38) is only a systematic review, which lacks rigorous and reproducible metaanalyses. In a meta-analysis of DSVPTC published in July 2016, Malandrino et al. (39) searched in PubMed and found only ten studies for meta-analyses. The authors, however, included studies in which follicular variant or encapsulated variant were also included in the control group $(40,41)$. The latter entity has recently been reclassified as a benign lesion (42). In addition, two large cohort studies by Kazaure et al. (11) and Kuo et al. (43) both selected patients from the Surveillance, Epidemiology and End Results (SEER) database and can result in duplicating data and bias the meta-analysis results. In our current study, we searched five large electronic databases (PubMed, ISI, Scopus, WHO GHL and VHL) and finally included 16 studies for meta-analyses, which enhance the statistical power of our analyses. Additionally, to strengthen the integrity of our analyses, we only selected studies that included cPTCs only as control group and the definition for DSVPTC was clearly described in most of the included studies. Finally, the genetic backgrounds of DSVPTC and cPTC were carefully investigated in our study to better highlight and confirm the different nature of these two variants. 
Our results demonstrated that DSVPTC displays more aggressive behaviors than CPTC, and this variant should thus be regarded as an aggressive variant of PTCs and classified as ATA intermediate- or high-risk group (37). Total thyroidectomy in conjunction with prophylactic central with/without lateral neck dissection followed by radioiodine ablation could be treatment of choice in management strategies for DSVPTCs because of the high propensity for vascular invasion, extrathyroidal extension, lymph node metastasis and distant metastasis. Hence, correct preoperative diagnosis is very important. Ultrasonography is a useful tool for suggesting DSVPTC due to extensive calcification and psammoma bodies in DSVPTCs and can help prompt fine needle aspiration (FNA), especially in young patients $(44,45,46)$. FNA showing numerous psammoma bodies, lymphoplasmacytic background and an absence or lack of characteristic nuclear features of PTC could suggest a diagnosis of DSVPTC (47). On histopathological examination, DSVPTC shows marked squamous metaplasia, numerous psammoma bodies, extensive interstitial fibrosis and heavy lymphocytic infiltration (38).

The AJCC staging data of DSVPTCs and cPTCs were very limited and not included for meta-analysis. DSVPTCs were found to be associated with more advanced AJCC stage than cPTCs (7). Our study also showed that patients with DSVPTC are more likely to relapse than patients with cPTC and suggests a precise and long-term follow-up schedule for these patients. In several studies, the survival rate of DSVPTCs was high and not statistically different from cPTCs $(5,8$, $9,11)$. DSVPTCs tend to be found in younger patients in whom response to treatment is good, which can help to explain this finding. On the other hand, $\mathrm{Al}$ Qahtani et al. reported that patients with DSVPTC had a higher mortality rate than patients with CPTC (6). In the study by Malandrino et al., the authors found no significant difference in cancer-related death between DSVPTC and cPTC (OR: 1.34; 95\% CI: 0.762.38) (39). However, time-to-event outcomes are most appropriately analyzed using HR in systematic reviews and meta-analyses (15). In our current study, pooled HR for OS demonstrated that DSVPTC patients are associated with a compromised OS (HR: 1.89; 95\% CI: 1.36-2.62). This significant result, however, needs to be further confirmed in new studies as the number of included studies is quite small.

In our study, considerable amounts of heterogeneity were found among the several outcomes. This may stem from differences between the studies in how the authors defined DSVPTC and CPTC. The diagnostic criteria of cPTC are not provided in several studies (Table 1). CPTC is the most common histologic variant of PTC, and the histologic criteria for diagnosis of this variant are usually straightforward for thyroid pathologists and sometimes, therefore, were not mentioned in some studies. On the other hand, the diagnostic criteria for diagnosis of DSVPTC are well described in most of the included studies and resemble the criteria described by the WHO classification of malignant tumors (2), except for three studies which give no description (Table 1). Our study, however, is subject to another limitation in that some studies did not describe how they measured some of the clinicopathological characteristics, which could raise the heterogeneity among studies.

Because of the rarity of this variant, only a few studies compared the prevalence of BRAF mutations between DSVPTCs and cPTCs. In the study by Lim et al., the prevalence of BRAF mutations in DSVPTCs was significantly lower than that in cPTCs (25). On the other hand, RET/PTC rearrangements were reported to be the major events in DSVPTCs $(32,33)$. Joung et al. found that RET/PTC3 in DSVPTCs were associated with advanced stage and higher frequency of persistent disease (32). A few studies also found the association of RET/PTC3 in PTCs and advanced disease stage $(48,49)$. This relationship, however, remains controversial in the literature. The high prevalence of RET/PTC3 in DSVPTCs can help to explain why DSVPTCs usually occur in young patients. Unlike RET/PTC rearrangements, BRAF mutations have been shown to associate with aggressive clinical manifestations and poor outcomes in a number of studies and a thorough meta-analysis (50). However, BRAF mutations were not major events in DSVPTCs. This suggests that the biological aggressiveness of DSVPTCs might be related to a different molecular pathway.

In conclusion, our meta-analysis study demonstrated that DSVPTC is a rare variant of PTC with distinct clinicopathological and molecular characteristics and is commonly seen in young patients. This variant should be considered an aggressive variant of PTC, and appropriate treatments should be considered for these patients because of higher incidence of tumor invasion, metastasis, recurrence and mortality.

\footnotetext{
Supplementary data
}

This is linked to the online version of the paper at http://dx.doi.org/ 10.1530/EJE-16-0863. 


\section{Declaration of interest}

The authors declare that there is no conflict of interest that could be perceived as prejudicing the impartiality of the research reported.

\section{Funding}

This research did not receive any specific grant from any funding agency in the public, commercial or not-for-profit sector.

\section{Author contribution statement}

Huy Gia Vuong, Tetsuo Kondo, Lewis Hassell and Ryohei Katoh contributed to conceptualization, methodology, software, validation, formal analysis, investigation, resources, data curation, writing of the original draft, reviewing and editing the manuscript, visualization, supervision and project administration. Thong Quang Pham contributed to methodology, validation, formal analysis, investigation, resources, data curation, writing of the original draft, reviewing and editing the manuscript. Naoki Oishi, Kunio Mochizuki and Tadao Nakazawa contributed to methodology, resources, data curation, writing of the original draft, reviewing and editing the manuscript.

\section{References}

1 Kondo T, Ezzat S \& Asa SL. Pathogenetic mechanisms in thyroid follicular-cell neoplasia. Nature Reviews Cancer 20066 292-306. (doi:10.1038/nrc1836)

2 DeLellis RA, Lloyd RV, Heitz PU \& Eng C. World Health Organization Classification of Tumours. Pathology and Genetics of Tumours of Endocrine Organs. Lyon, France: IARC Press, 2004.

3 LiVolsi VA. Papillary thyroid carcinoma: an update. Modern Pathology 201124 S1-S9. (doi:10.1038/modpathol.2010.129)

4 Vickery AL, Carcangiu ML, Johannessen JV \& Sobrinho-Simoes M. Papillary carcinoma. Seminars in Diagnostic Pathology 19852 90-100.

5 Akaishi J, Sugino K, Kameyama K, Masaki C, Matsuzu K, Suzuki A, Uruno T, Ohkuwa K, Shibuya H, Kitagawa W et al. Clinicopathologic features and outcomes in patients with diffuse sclerosing variant of papillary thyroid carcinoma. World Journal of Surgery 201539 1728-1735. (doi:10.1007/s00268-015-3021-9)

6 Al-Qahtani KH, Al Asiri M, Tunio MAK, Aljohani NJ, Bayoumi Y \& AlShakwer W. Diffuse sclerosing variant papillary thyroid carcinoma: clinicopathological and treatment outcome analysis of 44 cases. Kuwait Medical Journal 201547230.

7 Falvo L, Giacomelli L, Andrea V, Marzullo A, Guerriero G \& de Antoni E. Prognostic importance of sclerosing variant in papillary thyroid carcinoma. American Surgeon 200672 438-444.

8 Regalbuto C, Malandrino P, Tumminia A, Le Moli R, Vigneri R \& Pezzino V. A diffuse sclerosing variant of papillary thyroid carcinoma: clinical and pathologic features and outcomes of 34 consecutive cases. Thyroid 201121 383-389. (doi:10.1089/thy.2010.0331)

9 Albareda M, Puig-Domingo M, Wengrowicz S, Soldevila J, Matias-Guiu X, Caballero A, Chico A \& De Leiva A. Clinical forms of presentation and evolution of diffuse sclerosing variant of papillary carcinoma and insular variant of follicular carcinoma of the thyroid. Thyroid 19988 385-391. (doi:10.1089/thy.1998.8.385)

10 Moreno Egea A, Rodriguez Gonzalez JM, Sola Perez J, Soria T \& Parrilla Paricio P. Clinicopathological study of the diffuse sclerosing variety of papillary cancer of the thyroid. Presentation of 4 new cases and review of the literature. European Journal of Surgical Oncology 199420 7-11.

11 Kazaure HS, Roman SA \& Sosa JA. Aggressive variants of papillary thyroid cancer: incidence, characteristics and predictors of survival among 43,738 patients. Annals of Surgical Oncology 201219 1874-1880. (doi:10.1245/s10434-011-2129-x)
12 Moher D, Liberati A, Tetzlaff J, Altman D \& PRISMA Group. Preferred reporting items for systematic reviews and meta-analyses: the PRISMA statement. PLoS Medicine 20096 e1000097. (doi:10.1371/journal. pmed.1000097)

13 Hozo SP, Djulbegovic B \& Hozo I. Estimating the mean and variance from the median, range, and the size of a sample. BMC Medical Research Methodology 20055 13. (doi:10.1186/1471-2288-5-13)

14 Borenstein M, Hedges LV, Higgins JP \& Rothstein HR. A basic introduction to fixed-effect and random-effects models for meta-analysis. Research Synthesis Methods 2010 1 97-111. (doi:10.1002/jrsm.12)

15 Tierney JF, Stewart LA, Ghersi D, Burdett S \& Sydes MR. Practical methods for incorporating summary time-to-event data into metaanalysis. Trials 20078 16. (doi:10.1186/1745-6215-8-16)

16 Higgins JP \& Thompson SG. Quantifying heterogeneity in a metaanalysis. Statistics in Medicine 200221 1539-1558. (doi:10.1002/ sim.1186)

17 Higgins JP, Thompson SG, Deeks JJ \& Altman DG. Measuring inconsistency in meta-analyses. BMJ 2003327 557-560. (doi:10.1136/ bmj.327.7414.557)

18 Hamilton WK \& Mizumoto A. MAVIS: Meta Analysis Via Shiny, 2015. Retrieved from http://cran.r-project.org/web/packages/ MAVIS/MAVIS.pdf.

19 Wells GA, Shea B, O'Connell D, Peterson JEA, Welch V, Losos M \& Tugwell P. The Newcastle-Ottawa Scale (NOS) for assessing the quality of nonrandomised studies in meta-analyses. Ottawa Hospital Research Institute 2012.

20 Balachandar S, La Quaglia M, Tuttle RM, Heller G, Ghossein RA \& Sklar CA. Pediatric differentiated thyroid carcinoma of follicular cell origin: prognostic significance of histologic subtypes. Thyroid 201626 219-226. (doi:10.1089/thy.2015.0287)

21 Fridman M, Lam AKy \& Krasko O. Characteristics of young adults of Belarus with post-Chernobyl papillary thyroid carcinoma: a long-term follow-up of patients with early exposure to radiation at the 30th anniversary of the accident. Clinical Endocrinology 201685 971-978. (doi:10.1111/cen.13137)

22 Khan DM, Manimaran D, Bharathi K, Tulasi Raman R, Hemanathan, Anuradha S, Srividya VVL \& Ramakrishna BA. Classical papillary thyroid carcinoma and its histopathological variants. Research Journal of Pharmaceutical, Biological and Chemical Sciences 20145200.

23 Kim SK, Park I, Woo J-W, Lee JH, Choe J-H, Kim JH \& Kim JS. Follicular and diffuse sclerosing variant papillary thyroid carcinomas as independent predictive factors of loco-regional recurrence: a comparison study using propensity score matching. Thyroid 201626 1077-1084. (doi:10.1089/thy.2016.0113)

24 Lam AKY \& Lo CY. Diffuse sclerosing variant of papillary carcinoma of the thyroid: a 35-year comparative study at a single institution. Annals of Surgical Oncology 200613 176-181.

25 Lim JY, Hong SW, Lee YS, Kim B-W, Park CS, Chang H-S \& Cho JY. Clinicopathologic implications of the BRAF(V600E) mutation in papillary thyroid cancer: a subgroup analysis of 3130 cases in a single center. Thyroid 201323 1423-1430. (doi:10.1089/ thy.2013.0036)

26 Nikiforov Y \& Gnepp DR. Pediatric thyroid cancer after the Chernobyl disaster. Pathomorphologic study of 84 cases (1991-1992) from the Republic of Belarus. Cancer 199474 748-766.

27 Soares J, Limbert E \& Sobrinho-Simoes M. Diffuse sclerosing variant of papillary thyroid carcinoma. A clinicopathologic study of 10 cases. Pathology, Research and Practice 1989185 200-206.

28 Koo JS, Shin E \& Hong SW. Immunohistochemical characteristics of diffuse sclerosing variant of papillary carcinoma: comparison with conventional papillary carcinoma. APMIS 2010118 744-752. (doi:10.1111/j.1600-0463.2010.02653.x)

29 Lin X, Finkelstein SD, Zhu B \& Silverman JF. Molecular analysis of multifocal papillary thyroid carcinoma. Journal of Molecular Endocrinology 200841 195-203. (doi:10.1677/JME-08-0063) 
30 Onder S, Ozturk Sari S, Yegen G, Sormaz IC, Yilmaz I, Poyrazoglu S, Sanli Y, Giles Senyurek Y, Kapran Y \& Mete O. Classic architecture with multicentricity and local recurrence, and absence of TERT promoter mutations are correlates of BRAF (V600E) harboring pediatric papillary thyroid carcinomas. Endocrine Pathology 201627 153-161. (doi:10.1007/s12022-016-9420-0)

31 Chou A, Fraser S, Toon CW, Clarkson A, Sioson L, Farzin M, Cussigh C, Aniss A, O’Neill C, Watson $\mathrm{N}$ et al. A detailed clinicopathologic study of ALK-translocated papillary thyroid carcinoma. American Journal of Surgical Pathology 201539 652-659. (doi:10.1097/PAS.0000000000000368)

32 Joung JY, Kim TH, Jeong DJ, Cho YY, Jang HW, Jung YY, Oh YL, Yim HS, Kim YL, Chung J et al. Diffuse sclerosing variant of papillary thyroid carcinoma:major genetic alterations and prognostic implications. Histopathology 201669 45-53. (doi:10.1111/his.12902)

33 Sheu S-Y, Schwertheim S, Worm K, Grabellus F \& Schmid KW. Diffuse sclerosing variant of papillary thyroid carcinoma: lack of BRAF mutation but occurrence of RET/PTC rearrangements. Modern Pathology 200720 779-787.

34 Chung KW, Chang MC, Noh DY, Oh SK, Choe KJ \& Youn YK. RET oncogene expression of papillary thyroid carcinoma in Korea. Surgery Today 200434 485-492.

35 Musholt TJ, Musholt PB, Khaladj N, Schulz D, Scheumann GF \& Klempnauer J. Prognostic significance of RET and NTRK1 rearrangements in sporadic papillary thyroid carcinoma. Surgery 2000 128 984-993.

36 Bae JS, Kim Y, Jeon S, Kim SH, Kim TJ, Lee S, Kim MH, Lim DJ, Lee YS \& Jung CK. Clinical utility of TERT promoter mutations and ALK rearrangement in thyroid cancer patients with a high prevalence of the BRAF V600E mutation. Diagnostic Pathology 20161121. (doi:10.1186/s13000-016-0458-6)

37 Haugen BR, Alexander EK, Bible KC, Doherty GM, Mandel SJ, Nikiforov YE, Pacini F, Randolph GW, Sawka AM, Schlumberger $\mathrm{M}$ et al. 2015 American thyroid association management guidelines for adult patients with thyroid nodules and differentiated thyroid cancer: the American thyroid association guidelines task force on thyroid nodules and differentiated thyroid cancer. Thyroid 201626 1-133. (doi:10.1089/thy.2015.0020)

38 Pillai S, Gopalan V, Smith RA \& Lam AK. Diffuse sclerosing variant of papillary thyroid carcinoma-an update of its clinicopathological features and molecular biology. Critical Reviews in Oncology/Hematology 201594 64-73. (doi:10.1016/j.critrevonc.2014.12.001)

39 Malandrino P, Russo M, Regalbuto C, Pellegriti G, Moleti M, Caff A, Squatrito $S \&$ Vigneri R. Outcome of the diffuse sclerosing variant of papillary thyroid cancer: a meta-analysis. Thyroid 201626 1285-1292. (doi:10.1089/thy.2016.0168)
40 Chow SM, Chan JK, Law SC, Tang DL, Ho CM, Cheung WY, Wong IS \& Lau WH. Diffuse sclerosing variant of papillary thyroid carcinoma-clinical features and outcome. European Journal of Surgical Oncology 200329 446-449.

41 Koo JS, Hong S \& Park CS. Diffuse sclerosing variant is a major subtype of papillary thyroid carcinoma in the young. Thyroid 200919 1225-1231. (doi:10.1089/thy.2009.0073)

42 Nikiforov YE, Seethala RR, Tallini G, Baloch ZW, Basolo F, Thompson LD, Barletta JA, Wenig BM, Al Ghuzlan A, Kakudo K et al. Nomenclature revision for encapsulated follicular variant of papillary thyroid carcinoma: a paradigm shift to reduce overtreatment of indolent tumors. JAMA Oncology 20162 1023-1029. (doi:10.1001/ jamaoncol.2016.0386)

43 Kuo C-S, Tang K-T, Lin J-D, Yang A-H, Lee C-H \& Lin H-D. Diffuse sclerosing variant of papillary thyroid carcinoma with multiple metastases and elevated serum carcinoembryonic antigen level. Thyroid 201222 1187-1190. (doi:10.1089/thy.2011.0361)

44 Lee JH, Shin JH, Lee H-W, Oh YL, Hahn SY \& Ko EY. Sonographic and cytopathologic correlation of papillary thyroid carcinoma variants. Journal of Ultrasound in Medicine 201534 1-15. (doi:10.7863/ ultra.34.1.1)

45 Lin-xian YUE, Yi MA, Li-qiang D, Zhi-qing CAI \& Shi-guang W. Ultrasound features of diffuse sclerosing variant of papillary carcinoma of the thyroid. Chinese Journal of Ultrasonography $2009 \mathbf{1 8}$ 783-785.

46 Lee JY, Shin JH, Han B-K, Ko EY, Kang SS, Kim JY, Oh YL \& Chung JH. Diffuse sclerosing variant of papillary carcinoma of the thyroid: imaging and cytologic findings. Thyroid 200717 567-573.

47 Takagi N, Hirokawa M, Nobuoka Y, Higuchi M, Kuma S \& Miyauchi A. Diffuse sclerosing variant of papillary thyroid carcinoma: a study of fine needle aspiration cytology in 20 patients. Cytopathology 201425 199-204. (doi:10.1111/cyt.12076)

48 Nikiforov YE, Steward DL, Robinson-Smith TM, Haugen BR, Klopper JP, Zhu Z, Fagin JA, Falciglia M, Weber K \& Nikiforova MN. Molecular testing for mutations in improving the fine-needle aspiration diagnosis of thyroid nodules. Journal of Clinical Endocrinology and Metabolism 200994 2092-2098. (doi:10.1210/jc.2009-0247)

49 Romei C, Ciampi R, Faviana P, Agate L, Molinaro E, Bottici V, Basolo F, Miccoli P, Pacini F, Pinchera A et al. BRAFV600E mutation, but not $\mathrm{RET} / \mathrm{PTC}$ rearrangements, is correlated with a lower expression of both thyroperoxidase and sodium iodide symporter genes in papillary thyroid cancer. Endocrine-Related Cancer 200815 511-520. (doi:10.1677/ERC-07-0130)

50 Li C, Lee KC, Schneider EB \& Zeiger MA. BRAF V600E mutation and its association with clinicopathological features of papillary thyroid cancer: a meta-analysis. Journal of Clinical Endocrinology and Metabolism 201297 4559-4570. (doi:10.1210/jc.2012-2104)

Received 17 October 2016

Revised version received 29 December 2016

Accepted 17 January 2017 